\title{
SAMU/192 e a Abordagem Pré-Hospitalar do Infarto Agudo do Miocárdio no Brasil: Esperança para o Paciente ou Mais Uma Oportunidade Perdida?
}

SAMU-192 and the Prehospital Approach to Acute Myocardial Infarction in Brazil: Hope for Patients or One More Missed Opportunity?

Antonio Luiz da Silva Brasileiro

Instituto Nacional de Cardiologia, Hospital Barra D'Or - Rio de Janeiro, RJ

\section{Introdução}

A partir da publicação do estudo realizado pelo Gruppo Italiano per lo Studio della Sopravvivenza nell'Infarto miocardico (GISSI), em $1986^{1}$, os pacientes acometidos por infarto agudo do miocárdio (IAM) passaram a contar com uma nova e poderosa arma capaz de aumentar suas chances de sobrevivência em até $50 \%$, quando realizada em tempo hábil: a trombólise.

Desde então, em todo o mundo, várias estratégias foram criadas com o objetivo de reduzir o tempo decorrido entre o início dos sintomas e o tratamento, o chamado delta T. Surgiram "Protocolos de Dor Torácica", recomendações para realização da trombólise já na Emergência, campanhas públicas de orientação para a população, além de inúmeras reportagens educativas sobre o tema, mas nenhuma delas tinha maior potencial que a "Trombólise Pré-Hospitalar", ou seja, a trombólise iniciada ainda na ambulância. Uma metaanálise publicada em $2000^{2}$ confirmava os benefícios dessa estratégia. Entretanto, três importantes entraves impediam sua disseminação: 1) impossibilidade de diagnóstico confiável realizado pelas equipes das ambulâncias, geralmente formadas por não-cardiologistas ou por paramédicos; 2) dificuldades decorrentes da infusão intravenosa da droga em ambiente não-hospitalar; e 3) conduta indicada para os pacientes com contra-indicação para trombólise e para aqueles sem resposta ao trombolítico.

O primeiro entrave foi superado graças ao progresso da tecnologia de transmissão digital de dados, hoje corriqueira, permitindo o laudo do eletrocardiograma on line por um cardiologista à distância. O segundo foi resolvido pelo desenvolvimento ${ }^{3}$ e pela entrada no mercado de novos trombolíticos de administração em bolo, facilitando o uso da droga no espaço restrito da ambulância. O último entrave foi resolvido pela integração com centros terciários regionais de intervenção, capacitados para a realização de angioplastia de resgate ${ }^{4}$.

\section{Palavras-chave}

Infarto do miocárdio, atendimento de emergência pré-hospitalar, unidades móveis de emergência, primeiros socorros.

Correspondência: Luiz da Silva Brasileiro •

Av. Ayrton Senna, 2150/212 - bloco H - 22775-000 - Rio de Janeiro, RJ

E-mail: abrasileiro@cardiol.br

Artigo recebido em 3/01/07 ; aceito em 3/01/07.

\section{Um novo conceito: a abordagem pré- hospitalar}

O aumento exponencial da importância da angioplastia primária, demonstrando clara superioridade como estratégia de reperfusão para pacientes com apresentação tardia ${ }^{5}$, para pacientes de alto risco $^{6,7}$ e, evidentemente, para aqueles com maior risco de sangramentos, levou à substituição do conceito básico de "Trombólise Pré-Hospitalar" pelo conceito mais abrangente de "Abordagem Pré-Hospitalar do IAM", que inclui:

- diagnóstico precoce e confiável do IAM, auxiliado por teleconsultoria;

- integração, sempre que viável, com centros regionais terciários de intervenção;

- tomada precoce de decisão, ainda na ambulância, quanto à melhor estratégia de reperfusão, com imediata remoção do paciente para o centro terciário quando indicado por protocolo baseado nas melhores evidências ${ }^{8}$ (fig. 1);

- início pré-hospitalar do tratamento adjuvante adequado para cada caso.

Atualmente essa é a principal estratégia de atendimento ao IAM na Europa e com rápido crescimento nos Estados Unidos. Um estudo recentemente publicado ${ }^{9}$ comprovou que essa abordagem pode conseguir redução de 60 minutos do delta T e de $30 \%$ da mortalidade, mesmo quando o atendimento é realizado por enfermeiros, sem a presença de médicos.

Nas regiões em que não existam centros capazes de realizar intervenções coronarianas emergenciais (angioplastia primária ou de resgate) dentro de adequados padrões de qualidade, deve ser estimulada a realização de trombólise em todos os pacientes sem contra-indicação.

\section{SAMU/192 e a abordagem pré-hospitalar do infarto no Brasil}

Apesar dos inúmeros progressos nessa área, continua muito reduzido o número de pacientes com IAM que, no Brasil, recebem qualquer terapia de reperfusão. Na maior parte do país, não existem centros de intervenção, estando a angioplastia disponível, quase que exclusivamente, nos grandes centros das regiões Sul e Sudeste. Mesmo nessas regiões, porém, poucos pacientes têm acesso a esses procedimentos na rede pública, o que demonstra que a trombólise deverá ser, por muito tempo, a única estratégia de 


\section{ALGORITMO DE ABORDAGEM PRÉ-HOSPITALAR BASEADO NAS DIRETRIZES DA SBC / ESC / ACC / AHA}

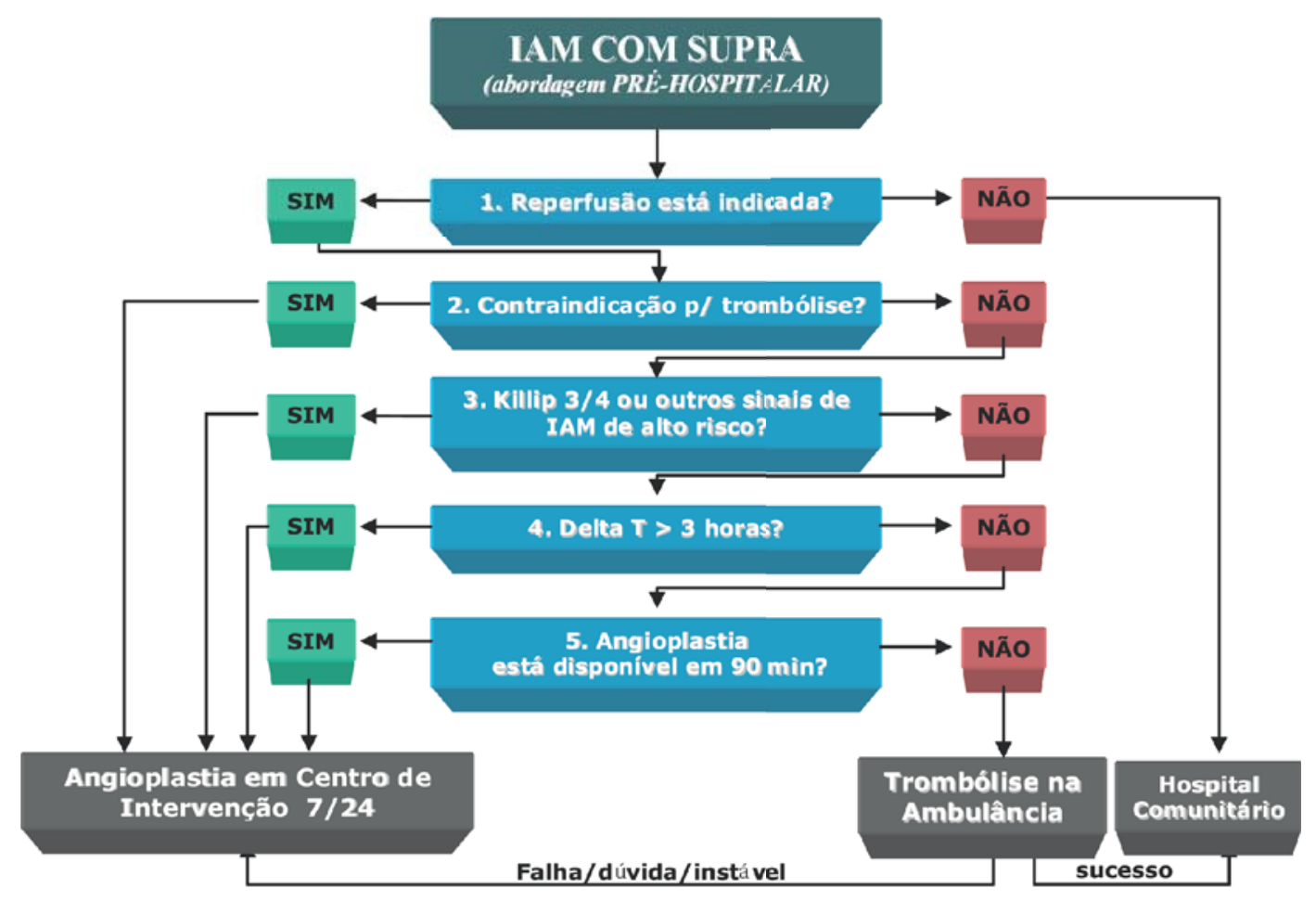

Fig. 1 - Figura demonstrando algoritmo que, utilizando as recomendações das principais diretrizes, pode orientar os profissionais envolvidos no atendimento préhospitalar do paciente com infarto agudo do miocárdio (IAM) na tomada de decisão quanto à melhor estratégia de reperfusão a ser escolhida para cada paciente atendido; $7 / 24=7$ dias por semana, 24 horas por dia.

reperfusão disponível. Dados não-publicados fornecidos pela indústria farmacêutica nos dão conta da baixa freqüência de utilização de trombolíticos, demonstrando que, para a maioria da população brasileira, a era da reperfusão, iniciada há mais de 20 anos, ainda não chegou.

Há, entretanto, uma nova esperança. O Ministério da Saúde, por meio da portaria 1.864, criou, em 29 de setembro de 2003, o Serviço de Atendimento Móvel de Urgência, o SAMU/192, com objetivos ambiciosos. Dados obtidos no site do Ministério da Saúde ${ }^{10}$ revelam que o SAMU já atende uma população de 81 milhões de brasileiros em 817 municípios de 25 Estados, utilizando mais de mil ambulâncias equipadas, além de "ambulanchas" e helicópteros. É, entretanto, frustrante constatar que, mais de três anos após sua implementação, o SAMU/192 não utilize rotineiramente a trombólise préhospitalar. É decepcionante constatar que em seu I Congresso Nacional ${ }^{11}$, ocorrido durante quatro dias em março de 2006, em Brasília, não constasse do programa do evento o tema "Atendimento do IAM". Tamanho investimento de dinheiro público somente se justifica se o sistema for capaz de oferecer à população atendimento de qualidade a suas principais causas de morbidade e mortalidade, ou seja, as emergências cardiovasculares. As estatísticas do próprio SAMU demonstram que estas constituem sua principal causa de atendimento, apontando para um enorme potencial para a aplicação dos conceitos atuais de "Abordagem Pré-Hospitalar do IAM".

Não bastam boas intenções e muitas ambulâncias. É necessário que o SAMU mantenha em seus quadros profissionais qualificados, Programa de Treinamento e Educação Continuada em Emergências Cardiológicas, protocolo atualizado de atendimento do IAM, integração com centros terciários regionais, além de rígido sistema de controle de qualidade. Além de centrais telefônicas que funcionem. Somente assim o SAMU estará cumprindo sua missão. A Sociedade Brasileira de Cardiologia - SBC não se negará a participar desse esforço, oferecendo uma "Diretriz de Abordagem Pré-Hospitalar do IAM" com a participação dos principais especialistas brasileiros no assunto.

Com a palavra as autoridades responsáveis pelo SAMU/192.

\section{Potencial Conflito de Interesses}

Declaro não haver conflitos de interesses pertinentes. 


\section{Ponto de Vista}

\section{Referências}

1. Gruppo Italiano per lo Studio della Streptochinasi nell'Infarto Miocardico (GISSI). Effectiveness of intravenous thrombolytic treatment in acute myocardial infarction. Lancet. 1986;1:397-401.

2. Morrison LJ, Verbeek PR, McDonald HC, Sawadsky BV, Cook DJ. Mortality and prehospital thrombolysis for acute myocardial infarction: a meta-analysis. JAMA. 2000; 283 (20): 2686-92.

3. Assessment of the Safety and Efficacy of a New Thrombolytic (ASSENT 2) Investigators, van de Werf F, Adgey J, Ardissino D, Armstrong PW, Aylward $P$, Barbash G, et al. Single-bolus tenecteplase compared with front-loaded alteplase in acute myocardial infarction double-blind randomised trial. Lancet. 1999; 354 (9180): 716-22.

4. Gershlick AH, Stephens Loyd A, Hughes S, Abrams KR, Stevens SE, Uren NG, REACT Trial Investigators, et al. Rescue angioplasty after failed thrombolytic therapy for acute myocardial infarction. N Engl J Med. 2005; 353 (26): 2758-68.

5. Steg PG, Bonnefoy E. Impact of time to treatment on mortality after prehospital thrombolysis. Circulation. 2003; 108 (23): 2851-6.

6. Hochman JS, Sleeper LA, Webb JG, Sanborn TA, White HD, Talley JD, et al. Early revascularization in acute myocardial infarction complicated by cardiogenic shock. SHOCK Investigators Should we Emergently Revascularize Occluded Coronaries for Cardiogenic Shock. N Engl J Med.
1999; 341 (9): 625-34.

7. Wu AH, Parsons L, Every NR, Bates ER. Second National Registry of Myocardial Infarction. Hospital outcomes in patients presenting with congestive heart failure complicating acute myocardial infarction: a report from the Second National Registry of Myocardial Infarction (NRMI-2). J Am Coll Cardiol. 2002; 40: 1389-94.

8. Antman EM, Anbe DT, Armstrong PW, Bates ER, Green LA, Hand M, et al. ACC/AHA guidelines for the management of patients with ST-elevation myocardial infarction: a report of the American College of Cardiology/ American Heart Association Task Force on Practice Guidelines. Circulation. 2004; 110 (9): e82-292.

9. Bjorklund E. Pre-hospital thrombolysis delivered by paramedics is associated with reduced time delay and mortality in ambulance-transported real-life patients with ST-elevation myocardial infarction. Eur Heart J. 2006; 27 (10): 1146-52.

10. Ministério da Saúde [homepage na internet]. Aplicações, notícias [citado 2007 janeiro 02]. Disponível em http://portal.saude.gov.br/portal/aplicacoes/ noticias/noticias_detalhe.cfm?co_seq_noticia $=28267$

11. Ministério da Saúde [homepage na internet]. Disponível em url: http://portal. saude.gov.br/portal/aplicacoes/noticias/noticias detalhe.cfm?co seq noticia $=24562$ 